\title{
A INTERFERÊNCIA DOS FATORES PSICOLÓGICOS NA HIPERTENSÃO ARTERIAL
}

THE INTERFERENCE OF PSYCHOLOGICAL FACTORS IN ARTERIAL HYPERTENSION

PINTO, Thaís Santos Bezerra ${ }^{1}$, PINTO, Antonione Santos Bezerra², TORRES, Amanda Azevedo ${ }^{3}$, FIRMINO, Brunna da Silva ${ }^{4}$, SOUSA, André Luca Araujo de ${ }^{4^{*}}$, BEZERRA, Sabrina Lisboa ${ }^{5}$

\author{
${ }^{1}$ Faculdade Santo Agostinho, Teresina -PI, Brasil. \\ 2 Universidade Federal do Ceará, Parnaíba -PI, Brasil. \\ 3Universidade Federal do Piauí, Parnaíba -PI, Brasil. \\ ${ }^{4}$ Universidade Estadual do Piauí, Parnaíba -PI, Brasil. \\ 5Universidade Estadual do Rio Grande do Norte, Mossoró -RN, Brasil. \\ *a.lucaaraujo10@gmail.com
}

\section{RESUMO}

A hipertensão arterial é condição clínica multifatorial caracterizada por elevação sustentada dos níveis pressóricos. São atribuídos como causas para a elevação da pressão arterial fatores constitucionais, ambientais e fatores ligados ao grupo social ao qual o sujeito pertence. Ainda que os fatores genéticos sejam conhecidos como importantes na etiologia da Hipertensão Arterial Sistêmica. O objetivo da pesquisa quantitativa foi identificar a interferência dos fatores psicológicos no agravamento da hipertensão. Participaram 61 pacientes hipertensos acompanhados na Unidade Básica de Saúde Vereador Durval Costa, Mossoró-RN. A coleta de dados ocorreu em 3 etapas: a) Entrevista com coleta de histórico clínico e pessoal da HAS; b) Inventário de Ansiedade Traço-Estado [IDATE]; e c) Aplicação do Inventário de Sintomas de Stress para Adultos de Lipp (ISSL). 100\% dos pacientes descrevem a "preocupação" como essencial pela frequente elevação da Pressão Arterial $(p=0,002)$ e $64 \%$ referem-se aos conflitos emocionais existentes na família $(p=0,018)$. Em relação aos escores de ansiedade, existem diferenças estatísticas entre as pessoas que possuem 
PINTO, Thaís Santos Bezerra, PINTO, Antonione Santos Bezerra, TORRES, Amanda Azevedo, FIRMINO, Brunna da Silva, SOUSA, André Luca Araujo de, BEZERRA, Sabrina Lisboa.

aumento frequente dos níveis pressóricos. Portanto, o objetivo de identificar a relação entre escores de ansiedade, estresse e a prevalência de conflitos emocionais com a elevação frequente da pressão arterial foi alcançado.

Palavras-chave: Hipertensão, Ansiedade, Stress, Psicologia.

\section{ABSTRACT}

Arterial hypertension is a multifactorial clinical condition characterized by sustained elevation of blood pressure levels. Constitutional and environmental factors and factors linked to the social group to which the subject belongs are attributed as causes for the rise in blood pressure. Although genetic factors are known to be important in the etiology of Systemic Arterial Hypertension. The objective of the present quantitative work was to identify the interference of psychological factors in hypertension. It was performed with 61 hypertensive patients followed up at the Durval Costa Primary Health Care Unit, Mossoró-RN. The data collection took place in three stages:a)Interview with the clinical and personal history of $\mathrm{SAH} ; \mathrm{b})$ Inventory of Anxiety Trait-State[IDATE]; and c)Application of "Inventário de Sintomas de Stress de Lipp"(ISSL). The $100 \%$ of patients described the "worry" as essential in frequently increase of blood pressure $(\mathrm{p}=0,002)$ and $64 \%$ referred to emotional conflicts in the family $(p=0,018)$. About the anxiety scores, there was statistic differences between people who have frequent increase of blood pressure. Therefore, the goal of association between anxiety scores, stress and emotional conflicts prevalence with frequently increase of blood pressure was reached.

Keywords: Hypertension, Anxiety, Stress, Psychology.

\section{INTRODUÇÃO}

A hipertensão arterial é condição clínica multifatorial caracterizada por elevação sustentada dos níveis pressóricos $\geq 140$ e/ou $90 \mathrm{mmHg}$ conforme a VII Diretriz Brasileira de Hipertensão Arterial ${ }^{1}$.

São atribuídos como causas para a elevação da pressão arterial fatores constitucionais, ambientais e fatores ligados ao grupo social ao qual o sujeito 
PINTO, Thaís Santos Bezerra, PINTO, Antonione Santos Bezerra, TORRES, Amanda Azevedo, FIRMINO, Brunna da Silva, SOUSA, André Luca Araujo de, BEZERRA, Sabrina Lisboa.

pertence. Ainda que os fatores genéticos sejam conhecidos como importantes na etiologia da Hipertensão Arterial Sistêmica (HAS), estudos clínicos colocam que a influência de fatores psicológicos é relevante para sua origem e desenvolvimento².

Estudos relatam a influência de fatores psicológicos sobre o organismo humano, havendo, desde então, o reconhecimento de ser o coração o órgão mais sensível a essas reações ${ }^{3}$. Dentre esses fatores, pode-se perceber a ansiedade como responsável por importante influência² ${ }^{2}$.

O conceito de ansiedade não envolve um critério unitário, principalmente no contexto psicopatológico. Dessa forma, a ansiedade pode ser definida como um conjunto de manifestações somáticas - aumento da frequência cardíaca e respiratória, sudorese, tensão muscular, náusea, vazio no estômago, tontura - e psicológicas apreensão, alerta, inquietude, hipervigilância, dificuldade de concentração e de conciliação do sono ${ }^{4}$.

Os estados de ansiedade são caracterizados por sentimentos de apreensão e tensão, percebidos de forma consciente, acompanhados pela ativação do sistema nervoso autônomo como uma resposta psicológica à ameaça ao autoconceito. Assim, quando um tensor é percebido pelo indivíduo ocorrem alterações mediadas pelo hipotálamo, que resultam no aumento da pressão arterial ${ }^{5}$.

Além de outras causas como a alimentação, o estresse emocional também está relacionado à etiologia da $\mathrm{HAS}^{6}$. O estresse pode ser conceituado como uma resposta do organismo às ameaças positivas ou negativas, os sintomas podem ser fisiológicos como: sudorese, tensão muscular, insônia, taquicardia, hipertensão súbita; e psicológicos como: tristeza, apatia, irritabilidade e angústia ${ }^{7}$.

O estresse pode causar a intensificação da HAS e as características de personalidade do hipertenso produzem em grande parte sua reatividade cardiovascular perante os estressores vinculados à expressão ou inibição de emoções $^{8}$. As emoções específicas, hiper-reatividade cardiovascular e sua relação causal com a hipertensão arterial têm sido motivo de muitos estudos, porém, uma das dificuldades consiste na dúvida sobre as alterações no organismo serem decorrentes das modificações que a doença proporciona, ou se antecedem e explicam a HAS ${ }^{9}$.

A partir disso, objetivou-se estudar as variáveis psicológicas que podem contribuir para a ocorrência da elevação da pressão arterial. 
PINTO, Thaís Santos Bezerra, PINTO, Antonione Santos Bezerra, TORRES, Amanda Azevedo, FIRMINO, Brunna da Silva, SOUSA, André Luca Araujo de, BEZERRA, Sabrina Lisboa.

\section{MÉTODOS}

A pesquisa tem natureza quantitativa de caráter exploratório, visando oferecer informações sobre a interferência de fatores psicológicos na HAS. No cálculo para uma proporção de amostra mediante função em software Excel, utilizamos uma população de 337, correspondente às pessoas com Hipertensão Arterial Sistêmica (HAS) cadastrada e em acompanhamento na área 146 da Unidade Básica de Saúde Vereador Durval Costa, no Walfredo Gurgel, município de Mossoró. A obtenção do resultado amostral se deu para o cálculo de tamanho da amostra para proporção de 337 indivíduos, precisão de 5\% e nível de confiança de 95\%. A amostra obtida para esses parâmetros foi de 61 indivíduos.

Foram incluídos no estudo pacientes entre 45 a 75 anos, alfabetizados, de ambos os sexos e com hipertensão arterial essencial como doença básica, sem patologia secundária. Todos os pacientes entrevistados estavam em uso de medicação anti-hipertensiva. Como critérios de exclusão: pacientes que não possuíam condições físicas e cognitivas de responder aos instrumentos de coleta da pesquisa e com diagnóstico de problemas psiquiátricos para responder aos instrumentos.

A pesquisa foi aprovada no Comitê de Ética em Pesquisa sob o número 1.577.668. A coleta de dados ocorreu mediante leitura e assinatura do Termo de Consentimento Livre e Esclarecido (TCLE) com os participantes indicados, no qual foram esclarecidos os objetivos da pesquisa e o método proposto.

A coleta realizou-se em três etapas, foram elas: (1) o acompanhamento da pesquisadora, profissional de Psicologia, com entrevista, bem como o histórico clínico e pessoal da HAS; (2) Inventário de Ansiedade Traço-Estado (IDATE); e (3) Aplicação do Inventário de Sintomas de Stress para Adultos de Lipp (ISSL).

Quanto ao procedimento, consultou-se os pacientes no dia mensal do grupo de monitoramento aos pacientes com Hipertensão e/ou Diabetes, o HIPERDIA, e em visita domiciliar com a agente comunitária de saúde quanto à possibilidade de participarem do estudo. Caso não houvesse condição clínica impeditiva, a pesquisadora conversava com os pacientes, em sala de espera para a consulta médica, dando informações sobre a pesquisa e confirmando os critérios de inclusão e exclusão. Quando o paciente manifestava interesse e disponibilidade em participar, anotava-se seus dados para marcação da entrevista e preenchimento do IDATE e ISSL. 
PINTO, Thaís Santos Bezerra, PINTO, Antonione Santos Bezerra, TORRES, Amanda Azevedo, FIRMINO, Brunna da Silva, SOUSA, André Luca Araujo de, BEZERRA, Sabrina Lisboa.

A anamnese foi realizada em visita domiciliar na área de abrangência da pesquisa, em comum acordo com a família, sobre data e horário da entrevista. Os dados gerais do sujeito foram agrupados em: filiação, sexo, idade, escolaridade, situação ocupacional, religião e os aspectos psicológicos e emocionais que contribuíram para o desenvolvimento da Hipertensão Arterial e que ainda colaboravam para sua manutenção. O modelo utilizado da anamnese foi o "Exame Clínico Psicológico" que tem como objetivo a exploração, a identificação e o planejamento de estratégias terapêuticas relacionadas aos distúrbios psíquicos.

Após realização das entrevistas, foi agendada, com todos os pacientes, uma data para o preenchimento dos testes, na própria Unidade Básica de Saúde.

Esse estudo escolheu o IDATE como referência para estudar o construto ansiedade e encontrar evidências de que seja capaz de influenciar diretamente no aumento da pressão arterial. Esse instrumento não está incluído na lista do Conselho Federal de Psicologia (CFP), por não existir estudos suficientes de validade e normatização no Brasil, no entanto, observa-se que nas pesquisas internacionais o IDATE é o instrumento mais utilizado para avaliar ansiedade.

Constitui-se em instrumento de autoavaliação e apresenta como característica principal a mensuração de aspectos inespecíficos que permeiam as situações estressantes como, por exemplo, tensão, nervosismo, irritabilidade, preocupação, apreensão e outros. O IDATE apresenta dois instrumentos independentes: traço de ansiedade e estado de ansiedade. Caracteriza-se por mensurar uma tendência de ação baseada na percepção de situações como uma ameaça, o que tende a elevar a ansiedade. Cada inventário contém vinte itens que são respondidos em escala com quatro opções. De acordo com as pontuações obtidas, considera-se ansiedade-traço baixa, quando os valores se encontram entre 20 a 34 pontos; moderada, entre 35 a 49 pontos; elevada, 50 a 64 pontos; e muito elevada, 65 a 80 pontos. A escala traço de ansiedade consiste em 20 afirmações que solicitam que os sujeitos descrevam como geralmente se sentem. Os indivíduos respondem a cada item do IDATE fazendo uma autoavaliação numa escala de quatro pontos, avaliada de acordo com o crivo ${ }^{5}$.

Foi utilizado também, como instrumento da pesquisa, o Inventário de Sintomas de Stress para adultos de Lipp (ISSL), o qual permite um diagnóstico do estresse; em qual fase se encontra e se tal sintoma se torna aparente de forma física ou psicológica. 
PINTO, Thaís Santos Bezerra, PINTO, Antonione Santos Bezerra, TORRES, Amanda Azevedo, FIRMINO, Brunna da Silva, SOUSA, André Luca Araujo de, BEZERRA, Sabrina Lisboa.

O ISSL fornece uma medida objetiva da sintomatologia do estresse em jovens acima de 15 anos e adultos, é um instrumento que visa identificar de modo objetivo os sintomas de estresse apresentados pelo paciente, avaliando o tipo de sintomas presentes (somáticos ou psicológicos) e a fase de estresse em que 0 paciente se encontra. Sua aplicação leva aproximadamente 10 minutos e pode ser realizada individualmente ou em grupos de até 20 pessoas.

$O$ Instrumento é formado por três quadros referentes às fases do estresse. $O$ primeiro quadro, composto de 15 itens refere-se aos sintomas físicos ou psicológicos que a pessoa tenha experimentado nas últimas 24 horas. O segundo, composto de dez sintomas físicos e cinco psicológicos, está relacionado aos sintomas queixados no último mês. E o terceiro quadro, composto de 12 sintomas físicos e 11 psicológicos, refere-se a sintomas vivenciados nos últimos três meses. No total, o ISSL apresenta 37 itens de natureza somática e 19 psicológicas, sendo os sintomas muitas vezes repetidos, diferindo somente em sua intensidade e seriedade ${ }^{7}$.

Os dados foram expressos em média e desvio padrão, bem como valores mínimos, máximos, mediana, frequência simples e porcentagem através do programa estatístico SPSS (Armonk, NY: IBM Corp.) versão 22.0. Para variáveis contínuas, após análise dos pressupostos paramétricos, diferença estatística dos escores de ansiedade entre os grupos G1 (elevação da pressão arterial frequente) e G2 (elevação da pressão arterial não frequente) foram obtidas por Mann-Whitney. As categóricas associações significativas da elevação dos níveis pressóricos frente às diferentes variáveis estudadas foram obtidas através Qui-quadrado e Exato de Fisher. Este último utilizado sempre quando verificado valores de frequência esperada inferiores a 5. Valores de $p<0,05$ foram considerados significativos.

A pesquisadora do presente estudo tem formação em Psicologia, tendo a autonomia necessária para aplicação, correção e arquivamento dos testes psicológicos. Que serão armazenados de forma sigilosa, na instituição formadora (UERN) em um período de cinco anos.

\section{RESULTADOS}

\section{Análise descritiva}

Atendendo aos objetivos do estudo, traçou-se um perfil sociodemográfico da amostra para a obtenção dos dados gerais dos pacientes com hipertensão arterial, 
PINTO, Thaís Santos Bezerra, PINTO, Antonione Santos Bezerra, TORRES, Amanda Azevedo, FIRMINO, Brunna da Silva, SOUSA, André Luca Araujo de, BEZERRA, Sabrina Lisboa.

cadastrados na Unidade Básica Vereador Durval Costa, da área 146, no município de Mossoró - RN.

A Tabela 1 evidencia a estatística descritiva sociodemográfica. A amostra estudada tem entre 45 a 75 anos, sendo que a maior concentração de sujeitos hipertensos está na faixa situada acima de 50 anos (73,8\%). Há predominância significativa do sexo feminino (82\%) sobre o masculino (18\%).

Em relação à escolaridade, grande parte concluiu o ensino fundamental $(50,8 \%)$, seguida daqueles com $2^{\circ}$ grau completo (41\%). O estado civil predominante é de pessoas casadas, constituindo $52,5 \%$ da população, seguido de pessoas solteiras $27,9 \%$. Em relação à inserção no mercado de trabalho, $72,5 \%$ são aposentados.

Tabela 1

Estatística descritiva sociodemográfico dos pacientes com Hipertensão Arterial, cadastrados na Unidade Básica Vereador Durval Costa, da área 146, município de Mossoró- RN

\begin{tabular}{|c|c|c|}
\hline Variáveis & Frequência & $\%$ \\
\hline \multicolumn{3}{|l|}{ Escolaridade } \\
\hline Fundamental & 31 & 50,8 \\
\hline Médio & 25 & 41,0 \\
\hline Superior & 05 & 8,2 \\
\hline \multicolumn{3}{|l|}{ Sexo } \\
\hline Masculino & 11 & 18,0 \\
\hline Feminino & 50 & 82,0 \\
\hline \multicolumn{3}{|l|}{ Estado civil } \\
\hline Solteiro & 17 & 27,9 \\
\hline Casado & 32 & 52,5 \\
\hline Viúvo & 12 & 19,7 \\
\hline \multicolumn{3}{|l|}{ Trabalha } \\
\hline Sim & 17 & 27,9 \\
\hline Não & 44 & 72,1 \\
\hline \multicolumn{3}{|l|}{ Idade } \\
\hline Até 50 anos & 16 & 26,2 \\
\hline Acima de 50 & 45 & 73,8 \\
\hline Média \pm desvio padrão & \multicolumn{2}{|c|}{$59,24 \pm 8,79$} \\
\hline Mínimo - Máximo & \multicolumn{2}{|c|}{$45-75$} \\
\hline \multicolumn{3}{|l|}{ Religião } \\
\hline Católico & 39 & 63,9 \\
\hline Evangélico & 14 & 23,0 \\
\hline Outros & 08 & 13,1 \\
\hline
\end{tabular}


PINTO, Thaís Santos Bezerra, PINTO, Antonione Santos Bezerra, TORRES, Amanda Azevedo, FIRMINO, Brunna da Silva, SOUSA, André Luca Araujo de, BEZERRA, Sabrina Lisboa.

A Tabela 2 evidencia que os conflitos emocionais citados pelos pacientes hipertensos foram "questões familiares" (82,0\%) e "preocupação" (45,9\%). Dos participantes que apresentaram níveis elevados de estresse, a fase crítica e perigosa foi a que mais prevaleceu entre os participantes, com 59,0\%. Verificou-se que $29,5 \%$ da amostra não apresentou sintomas de estresse. Quanto aos níveis pressóricos, $41 \%$ tiveram elevação frequente.

Tabela 2

Estatística descritiva dos aspectos psicológicos de pacientes com Hipertensão Arterial, cadastrados na Unidade Básica Vereador Durval Costa, da área 146, município de Mossoró- RN

\begin{tabular}{lcc}
\multicolumn{1}{c}{ Variáveis } & Freq. & \% \\
\hline Preocupação & & \\
$\quad$ Sim & 50 & 82,0 \\
$\quad$ Não & 11 & 18,0 \\
Conflitos na familia & & \\
$\quad$ Sim & 28 & 45,9 \\
$\quad$ Não & 33 & 54,1 \\
Situação de stress & & \\
$\quad$ Crítica & 36 & 59,0 \\
$\quad$ Descontrole & 05 & 8,2 \\
$\quad$ Intermediário & 02 & 3,3 \\
$\quad$ Sem sintomas & 18 & 29,5 \\
Elevação da Pressão Arterial & & \\
$\quad$ Frequentemente & 25 & 41,0 \\
$\quad$ Não frequente & 36 & 59,0 \\
\hline
\end{tabular}

\section{Análise das diferentes variáveis sociais associadas ao aumento da pressão arterial}

Como mostrado na Tabela 3, quando se associa HAS com as variáveis sociais, a idade tem influência significativa com a incidência da elevação pressórica que se mostrou mais evidente na faixa etária acima de 50 anos, apresentando-se como índice mais frequente $(56,0 \%)$. 
PINTO, Thaís Santos Bezerra, PINTO, Antonione Santos Bezerra, TORRES, Amanda Azevedo, FIRMINO, Brunna da Silva, SOUSA, André Luca Araujo de, BEZERRA, Sabrina Lisboa.

Tabela 3

Valores de frequência simples (\%) da elevação da pressão arterial frente às diferentes variáveis sociais

\begin{tabular}{|c|c|c|c|}
\hline \multirow[b]{2}{*}{ Variáveis } & \multicolumn{2}{|c|}{ Aumento da Hipertensão } & \multirow[b]{2}{*}{ p-valor } \\
\hline & $\begin{array}{c}\text { Frequente } \\
\mathrm{n}=25 \\
(41,0 \%)\end{array}$ & $\begin{array}{c}\text { Não } \\
\text { frequente } \\
\mathrm{n}=36 \\
(59,0 \%)\end{array}$ & \\
\hline \multicolumn{4}{|l|}{ Escolaridade } \\
\hline Fundamental & $12(48,0)$ & $19(52,8)$ & \multirow{3}{*}{0,922} \\
\hline Médio & $11(44,0)$ & $14(38,9)$ & \\
\hline Superior & $02(8,0)$ & $03(8,3)$ & \\
\hline \multicolumn{4}{|l|}{ Sexo } \\
\hline Masculino & $06(24,0)$ & $05(13,9)$ & \multirow{2}{*}{0,333} \\
\hline Feminino & $19(76,0)$ & $31(86,1)$ & \\
\hline \multicolumn{4}{|l|}{ Estado civil } \\
\hline Solteiro & $08(32,0)$ & $09(25,0)$ & \multirow{3}{*}{0,810} \\
\hline Casado & $12(48,0)$ & $20(55,6)$ & \\
\hline Viúvo & $05(20,0)$ & $07(19,4)$ & \\
\hline \multicolumn{4}{|l|}{ Trabalha } \\
\hline Sim & $06(24,0)$ & $11(30,6)$ & \multirow{2}{*}{0,574} \\
\hline Não & $19(76,0)$ & $25(69,4)$ & \\
\hline \multicolumn{4}{|l|}{ Idade } \\
\hline Até 50 anos & $11(44,0)$ & $05(13,9)$ & \multirow{2}{*}{$0,009 *$} \\
\hline Acima de 50 & $14(56,0)$ & $31(86,1)$ & \\
\hline \multicolumn{4}{|l|}{ Religião } \\
\hline Católico & $15(60,0)$ & $24(66,7)$ & \multirow{3}{*}{0,736} \\
\hline Evangélico & $07(28,0)$ & $07(19,4)$ & \\
\hline Outros & $03(12,0)$ & $05(13,9)$ & \\
\hline
\end{tabular}

* Significância estatística $(\mathrm{p}<0,05)$ 
PINTO, Thaís Santos Bezerra, PINTO, Antonione Santos Bezerra, TORRES, Amanda Azevedo, FIRMINO, Brunna da Silva, SOUSA, André Luca Araujo de, BEZERRA, Sabrina Lisboa.

\section{Análise dos aspectos psicológicos associados à elevação dos níveis pressóricos}

Tabela 4

Valores de frequência simples (\%) da elevação da pressão arterial frente às variáveis de interferência psicológica

\begin{tabular}{|c|c|c|c|}
\hline \multirow[b]{2}{*}{ Variáveis } & \multicolumn{2}{|c|}{ Aumento da Hipertensão } & \multirow[b]{2}{*}{ p-valor } \\
\hline & $\begin{array}{c}\begin{array}{c}\text { Frequente } \\
\mathrm{n}=25\end{array} \\
(41,0 \%)\end{array}$ & $\begin{array}{c}\text { Não } \\
\text { frequente } \\
\mathrm{n}=36 \\
(59,0 \%)\end{array}$ & \\
\hline \multicolumn{4}{|l|}{ Preocupação } \\
\hline Sim & $25(100,0)$ & $25(69,4)$ & \multirow{2}{*}{$0,002 *$} \\
\hline Não & $0(0,0)$ & $11(30,6)$ & \\
\hline \multicolumn{4}{|l|}{ Conflitos na família } \\
\hline Sim & $16(64,0)$ & $12(33,3)$ & \multirow{2}{*}{$0,018^{*}$} \\
\hline Não & $09(36,0)$ & $24(66,7)$ & \\
\hline \multicolumn{4}{|l|}{ Situação de Stress } \\
\hline Elevado (Crítico e descontrole) & $22(88,0)$ & $19(52,8)$ & \multirow{2}{*}{$0,004 * ¥$} \\
\hline Baixo (intermediário e sem sintomas) & $03(12,0)$ & $17(47,2)$ & \\
\hline
\end{tabular}

* Significância estatística $(\mathrm{p}<0,05)$.

¥ Categoria "Elevado" criado pela fusão dos grupos "Crítica" e "Descontrole" e categoria "Baixo" criado pela fusão dos grupos "Intermediários" e "Sem sintomas".

A Tabela 4 aponta que quando questionados sobre suas condições psicológicas $100 \%$ dos pacientes descrevem a "preocupação" como fator essencial do aumento frequente da pressão arterial $(p=0,002)$ e $64 \%$ referem-se aos conflitos emocionais existentes na família $(p=0,018)$. As associações relacionadas a conflitos familiares e preocupação foram consideradas significativas e positivas para a elevação dos níveis pressóricos. Dentre esses indivíduos com aumento frequente da pressão arterial, 88\% apresentaram sintomas compatíveis com a presença de estresse. Os resultados apontam a predominância de sintomas referentes à fase crítica e descontrole, quando considerados todos os sujeitos da amostra ( $p=0,004)$. 
PINTO, Thaís Santos Bezerra, PINTO, Antonione Santos Bezerra, TORRES, Amanda Azevedo, FIRMINO, Brunna da Silva, SOUSA, André Luca Araujo de, BEZERRA, Sabrina Lisboa.

Tabela 5

Valores de média \pm D.P dos escores de ansiedade para os grupos com elevação da pressão arterial "Frequente" $\mathrm{e}$ "Não frequente"

\begin{tabular}{lccccc}
\hline Variável & Grupos & Média \pm D.P. & Min-Máx. & Mediana & p-valor \\
\hline \multirow{2}{*}{ Ansiedade } & G1 $(\mathrm{n}=25)$ & $53,92 \pm 11,43$ & $28-73$ & 55,0 & \multirow{2}{*}{$0,004^{*}$} \\
& G2 $(\mathrm{n}=36)$ & $44,11 \pm 12,85$ & $28-70$ & 42,0 & \\
\hline
\end{tabular}

G1= Hipertensão "frequente"; G2= Não "frequente"; D.P.= Desvio padrão.

* Diferença estatística ( $<<0,05$ - Mann-Whitney).

Por fim, a Tabela 5 demonstra que quando verificado os escores de ansiedade, ao considerar os grupos frequente e não frequente quanto à elevação da pressão arterial, verificou-se que existem diferenças estatísticas quanto aos escores de ansiedade dos indivíduos hipertensos que possuem elevação da pressão arterial frequente, com média 53,92 $\pm 11,43$. Os resultados indicaram níveis altos de ansiedade, portanto, as associações encontradas entre indicadores de ansiedade (IDATE) e aumento da pressão arterial em pacientes hipertensos foram consideradas significativas.

\section{DISCUSSÃO}

Após analisar os resultados pode-se considerar que os objetivos propostos pelo presente estudo, de associar escores de ansiedade, estresse e aspectos emocionais com o aumento frequente dos níveis pressóricos foram atingidos e as hipóteses foram confirmadas. A amostra de hipertensos apresentou níveis altos de ansiedade e estresse.

A Sociedade Brasileira de Hipertensão cita que a idade tem relação direta e linear com a pressão arterial, sendo que na faixa etária acima de 65 anos, a prevalência de HAS é superior a $60 \%{ }^{1}$. Conforme citado nas VII Diretrizes Brasileiras de Hipertensão Arterial (2016), pesquisas evidenciam que 09 em cada 10 indivíduos a partir de 55 anos possivelmente desenvolverão hipertensão arterial durante sua vida, tornando-se um dos maiores problemas de saúde pública ${ }^{1}$.

De acordo com o estudo proposto por Mac Fadden e Ribeiro (1998) verificouse que a dificuldade da expressão afetivo-emocional, assim como a presença de ansiedade subjacente, pode agir sobre o sistema nervoso autônomo, favorecendo 
PINTO, Thaís Santos Bezerra, PINTO, Antonione Santos Bezerra, TORRES, Amanda Azevedo, FIRMINO, Brunna da Silva, SOUSA, André Luca Araujo de, BEZERRA, Sabrina Lisboa.

tensões hipertensivas transitórias que, ao longo do tempo, podem transformar-se em crises permanentes ${ }^{3}$.

No estudo "Mulheres e as influências afetivas no adoecimento hipertensivo" foi observada a influência das vivências pessoais e afetivas no desenvolvimento e na manifestação da HAS, seja de modo direto ou indireto ao próprio adoecimento, até mesmo percebendo a relação em outras mulheres hipertensas que convivem. O que leva a conjeturar que os indivíduos têm histórias anteriores ao diagnóstico, que podem interferir na forma como irão subjetivar e, desse modo, esta pesquisa aponta para a necessidade de que as questões subjetivas também sejam inseridas no tratamento, que possa ampliar a percepção deste que adoece, para que a experiência faça sentido ao sujeito ${ }^{10}$.

Os resultados da pesquisa indicaram níveis altos de preocupação e conflitos relacionados à família, esses dados indicam, como Ross e Souza, entre outros, que relacionam níveis baixos de percepção do suporte familiar com alta prevalência de transtornos ansiosos, transtornos de humor e outros problemas de saúde. Esses estudos assinalam a necessidade de mais atenção aos fatores psicossociais dos pacientes com hipertensão, demonstram a importância do apoio social, principalmente do suporte familiar, que se oferecido e percebido de forma adequada, pode se apresentar como um fator de proteção presente no processo saúde-doença. Já a inadequação do suporte familiar pode favorecer o comprometimento da saúde, compreendida de acordo com a definição da Organização Mundial da Saúde, como bem-estar físico, mental e social ${ }^{11,12}$.

Os conflitos familiares são considerados fatores de estresse social, que é um dos fatores ambientais de risco para o desenvolvimento da hipertensão e cardiopatias, dentre outros como, problemas financeiros, precárias condições de trabalho e moradia, dificuldades de transporte e violência. Estudos indicam que incluir a família em programas de atenção ao paciente hipertenso e à doença cardiovascular, pode facilitar a adesão ao tratamento e o maior controle da pressão arterial ${ }^{13}$.

O estudo "Raiva, Stress Emocional e Hipertensão: Um Estudo Comparativo" concluiu que uma comunicação clara é necessária para causar boas relações interpessoais e que falhas na comunicação decorrentes da excessiva inibição de raiva podem causar efeitos negativos na qualidade de vida e na área social da pessoa com hipertensão, incluindo tanto as situações familiares e conjugais, como os ocupacionais. Esse prejuízo é passível de ocasionar estresse, que por sua vez, 
PINTO, Thaís Santos Bezerra, PINTO, Antonione Santos Bezerra, TORRES, Amanda Azevedo, FIRMINO, Brunna da Silva, SOUSA, André Luca Araujo de, BEZERRA, Sabrina Lisboa.

poderá atuar acentuando a raiva. A saúde dessa pessoa também será afetada, pois se ocorrerem de forma exagerada, tanto a inibição da raiva quanto o estresse podem ocasionar aumento da reatividade cardiovascular, e assim, aumentar o impacto negativo, em todas as áreas da vida do indivíduo com hipertensão ${ }^{14}$.

Uma pesquisa desenvolvida na Inglaterra com 1.259 homens mostrou variações em pressão verificadas em hipertensos durante sessões experimentais em virtude do estresse psicológico. O estudo utilizou medidas estatísticas com procedimentos compensatórios que permitiram concluir que, embora oscilações pressóricas tivessem ocorrido, elas não poderiam justificar a reatividade cardiovascular verificada em inúmeros estudos. O estresse emocional teria sido o principal elemento desencadeador da reatividade ${ }^{8}$.

A importância de pesquisas sobre a influência do estresse emocional na reatividade cardiovascular deve ser enfatizada porque oferece a possibilidade de trabalhos na área da saúde direcionados à redução do estresse emocional, podem reduzir crises hipertensivas, muitas vezes desencadeadas por fatores estressantes da vida diária e também porque podem colaborar para o controle da hipertensão arterial sistêmica e para a prevenção de outras possíveis patologias associadas, diminuindo, então, o custo de medicamentos e assistência especializada ao sistema de saúde ${ }^{8}$.

O estudo sobre "A influência de fatores emocionais sobre a hipertensão arterial" concluiu que o risco de desenvolvimento da hipertensão arterial e a reatividade cardiovascular parecem ser influenciados por fatores psicológicos como impulsividade, hostilidade, estressores, ansiedade e raiva ${ }^{15}$. É possível também verificar em estudos que emoções como a ansiedade, quando bloqueadas, podem, por meio da influência que exercem no sistema nervoso autônomo, favorecer a crise hipertensiva em determinados pacientes com predisposição genética. Contudo, mais pesquisas são necessárias para melhor esclarecer essas relações ${ }^{15}$.

Os dados dessa amostra demonstraram um nível elevado ansiedade-traço (característica do indivíduo), que estão de acordo com alguns estudos, dentre eles, os de Vaz, citado por Pessotti (1978), que apresentam interações entre ansiedade e hipertensão, considerando a ansiedade como um dos fenômenos psíquicos presente no processo de saúde-doença ${ }^{16,17}$.

Segundo Faria, níveis aumentados de ansiedade e o consumo de álcool acima de três vezes por semana aumentaram, em aproximadamente, 5 (Odds ratio: 4,93) e 15 (Odds ratio: 15,19) vezes, respectivamente, as chances de o indivíduo 
PINTO, Thaís Santos Bezerra, PINTO, Antonione Santos Bezerra, TORRES, Amanda Azevedo, FIRMINO, Brunna da Silva, SOUSA, André Luca Araujo de, BEZERRA, Sabrina Lisboa.

apresentar hipertensão. Isso indica que o consumo de álcool e níveis aumentados de ansiedade intensificam as chances de o indivíduo ser hipertenso. Por outro lado, o uso de medicamentos antidepressivos diminuiu ligeiramente (Odds ratio: 0,15) a chance de acometimento por hipertensão, sugerindo que o controle do estado emocional exerce efeito protetor contra a hipertensão ${ }^{18}$.

Os resultados da presente pesquisa estão de acordo com as VII Diretrizes Brasileiras de Hipertensão $(2016)^{1}$, que identificam o estresse emocional e a ansiedade elevada como capaz de atuar no aumento transitório da reatividade cardiovascular e da pressão alta.

Com isso, esse estudo converge com pesquisas que indicam à ansiedade como uma das características psicológicas associadas à hipertensão arterial e que o tratamento demanda um apoio multiprofissional que considere aspectos relacionados ao controle da ansiedade, do estresse emocional e de outras características psicológicas dos pacientes.

\section{CONCLUSÃO}

É importante ressaltar que os aspectos psicológicos avaliados podem ser considerados como dois fatores presentes no processo saúde-doença, a ansiedade e o estresse como um fator de risco e a qualidade da relação familiar, como um fator de proteção. Contudo, existem outros fatores, de risco e de proteção, envolvidos no processo saúde-doença, tais como, alimentação, depressão, qualidade de vida, relações interpessoais, entre outros, que devem ser analisados em outras pesquisas.

O estudo contribuiu para estimular a complexidade do processo saúdedoença e que realmente a saúde deve ser compreendida como um estado biopsicossocial envolvendo a saúde física, psicológica e social. Dessa forma, fica evidente que a Psicologia na área da Saúde, pode colaborar para a compreensão do adoecimento e do fortalecimento de ações em promoção de saúde, colaborando no tratamento e acompanhamento dos pacientes com doenças crônicas, mas principalmente com trabalhos preventivos e políticas públicas que estimulem uma melhor qualidade de vida.

Os aspectos psicológicos avaliados ainda podem ser ampliados, destaca-se a necessidade da realização de pesquisas com outras interferências envolvidas na elevação da pressão arterial, tais como, verificar a relação dos níveis de ansiedade e 
PINTO, Thaís Santos Bezerra, PINTO, Antonione Santos Bezerra, TORRES, Amanda Azevedo, FIRMINO, Brunna da Silva, SOUSA, André Luca Araujo de, BEZERRA, Sabrina Lisboa.

estresse com o histórico pessoal do paciente, com amostras diferentes, estudos que comparem um grupo de hipertensos, com um grupo de pessoas que não tenha hipertensão (grupo controle) e estudos que controlem o uso de medicamentos.

Assim, recomenda-se cada vez mais a utilização de saberes variados, para que essas ações de atenção básica à saúde sejam mais eficazes e resolutivas, saberes tanto específicos de saúde como de outros campos de conhecimentos, compreendendo um exercício permanente de intersetorialidade e interdisciplinaridade. Então, é preciso ampliar o acompanhamento do paciente em função de particularidade das demandas que têm chegado aos equipamentos de saúde, demandas que conjugam as formas de adoecimento físico com as formas de sofrimento psíquico.

Portanto, a análise dos fatores psicológicos contribuintes para a ocorrência do aumento dos níveis pressóricos deve ser considerada a fim de que planos terapêuticos apropriados possam ser elaborados. Contudo, a assistência do Psicólogo dentro de uma equipe de saúde terá como objetivo oferecer suporte emocional, orientação e informação aos pacientes e seus familiares, visando sempre o fortalecimento do bem-estar emocional do indivíduo e das famílias. 
PINTO, Thaís Santos Bezerra, PINTO, Antonione Santos Bezerra, TORRES, Amanda Azevedo, FIRMINO, Brunna da Silva, SOUSA, André Luca Araujo de, BEZERRA, Sabrina Lisboa.

\section{REFERÊNCIAS}

1. Malachias MVB, Póvoa RMS, Nogueira AR, Souza D, Costa LS, Magalhães ME. $7^{\text {ạ }}$ Diretriz Brasileira de Hipertensão Arterial: Capítulo 2 - Diagnóstico e Classificação. Arq Bras Cardiol 2016; 107(Supl. 3):7-13.

2. Lessa I. Epidemiologia da hipertensão arterial. In: Lessa I. O adulto brasileiro e as doenças da modernidade: epidemiologia das doenças crônicas não transmissíveis. São Paulo: Editora Hucitec/Rio de Janeiro: ABRASCO; p. 77-96, 1998.

3. Mac Fadden MAJ, Ribeiro AV. Aspectos psicológicos e hipertensão essencial. Rev. Assoc. Med. Bras. [online]. 1998, vol.44, n.1, pp.410. https://doi.org/10.1590/S0104-42301998000100002.

4. Dratcu L, Lader M. Ansiedade: conceito, classificação e biologia. Jornal Brasileiro de Psiquiatria. 1993; 42(1): 19-32.

5. Spielberger CD, Biaggio A, Natalício LF. Inventário de ansiedade traço estado: manual de psicologia aplicada. Rio de Janeiro: CEPA, 1979.

6. Straub R. O. Psicologia da Saúde. Porto Alegre: Artmed Editora, 2005.

7. Lipp MEN. Manual de aplicação do inventário de sintomas de stress para adultos de Lipp. São Paulo: Casa do Psicólogo, 2000.

8. Lipp MEN. Controle do estresse e hipertensão arterial sistêmica. Revista Brasileira Hipertensão. 2007;14(2), p: 89-93.

9. Chaves EC, Cade NV. Efeitos da ansiedade sobre a pressão arterial em mulheres com hipertensão. Revista Latino-americana de Enfermagem. 2004;12(2), p: 162167.

10. Vaz AIA, Costa R. Mulheres e as influências afetivas no adoecimento hipertensivo. Psic. Revista São Paulo, 2015; 24(1), 83-106.

11. Ross C.E, Mirowsky J. Family Relationships, Social Support, and Subjective Life Expectancy. Journal of Health and Social Behavior. 2002; 43, 469-489. 
PINTO, Thaís Santos Bezerra, PINTO, Antonione Santos Bezerra, TORRES, Amanda Azevedo, FIRMINO, Brunna da Silva, SOUSA, André Luca Araujo de, BEZERRA, Sabrina Lisboa.

12. Souza MS, Baptista MN. Suporte familiar e saúde mental: evidência de validade baseada na relação com outras variáveis. Dissertação de Mestrado, Universidade São Francisco, Faculdade de Psicologia, Itatiba, São Paulo, 2007.

13. Tavares, M. Validade Clínica. Psico-USF, 2004; 8(2), 125-136.

14. Moxotó GFA, Malagris LEN. Raiva, Stress Emocional e Hipertensão: Um Estudo Comparativo. Psicologia: Teoria e Pesquisa. 2015; Abr-Jun 31(2), 221-227.

15. Fonseca FCA, Coelho RZ, Nicolato R, Malloy-Diniz LF, Filho, HCS. A influência de fatores emocionais sobre a hipertensão arterial. Jornada brasileira de psiquiatria. Rio de Janeiro, 2009; 58 (2).

16. Vaz, C. E. Característica de personalidade do hipertenso através de Rorschach Psico. Porto Alegre, 1990; 20(2), 33-43.

17. Pessotti, I. Ansiedade. São Paulo: EPU/EDUSP, 1978.

18. Faria FC, Faria BG. Avaliação de variáveis relacionadas ao risco à hipertensão em indivíduos atendidos pelo Programa de Saúde da Família. Enfermagem Brasil, 2016; 15(3). 